\title{
A pre seismic radio anomaly revealed in the area where the Abruzzo earthquake $(M=6.3)$ occurred on 6 April 2009
}

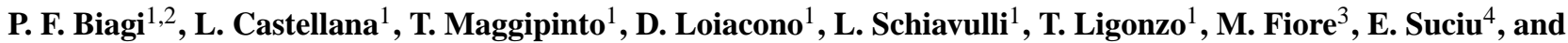 \\ A. Ermini ${ }^{5}$ \\ ${ }^{1}$ Department of Physics, University of Bari, Via Amendola 173, 70126 Bari, Italy \\ ${ }^{2}$ Inter-Department Centre for the Evaluation and Mitigation of the Volcanic and Seismic Risk, University of Bari, Italy \\ ${ }^{3}$ Elettronika s.r.l., Palo del Colle (Bari), Italy \\ ${ }^{4}$ Elettronika Research, Brasov, Romania \\ ${ }^{5}$ Department of Mechanical Engineering, University of Roma Tor Vergata, Via di Tor Vergata, 00133 Rome, Italy
}

Received: 7 July 2009 - Revised: 4 August 2009 - Accepted: 17 August 2009 - Published: 8 September 2009

\begin{abstract}
On 6 April 2009 a strong $\left(M_{w}=6.3\right)$ earthquake occurred in the Abruzzo region (central Italy). Since 1996, the intensity of CLT ( $f=189 \mathrm{kHz}$, Sicily, Italy), MCO $(f=216 \mathrm{kHz}$, France) and CZE $(f=270 \mathrm{kHz}$, Czech Republic) broadcast signals has been collected with a ten minutes sampling rate by a receiver operating in a place located about $13 \mathrm{~km}$ far from the epicenter. During March 2009, the old receiver was substituted with a new one able to measure, with one minute sampling rate, the intensity of five VLF signals and five LF signals radiated by transmitters located in different zones of Europe. The MCO and CZE transmitters mentioned above are included among them. From 31 March to 1 April the intensity of the MCO radio signal dropped and this drop was observed only in this signal. The possibility that the drop was connected to problems in the transmitter or in the receiver was investigated and excluded. So, the drop indicates a defocusing of the radiated signal. Since no particular meteorological situation along the path transmitter-receiver happened, the defocusing must be related to other causes, and a possibility is presented that it is a precursor of the Abruzzo earthquake.
\end{abstract}

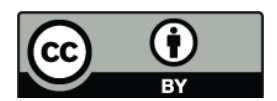

Correspondence to: P. F. Biagi

(biagi@fisica.uniba.it)

\section{Introduction}

The Abruzzo earthquake occurred near L'Aquila city (central Italy) on 6 April 2009 (UTC 01:32:39) producing damages and causalities. The magnitude $M_{w}$ was 6.3 ; a seismic sequence took place in the zone since the middle of January 2009; the aftershocks activity is still active and the epicentres are spread in a large area in North direction with respect to the location of the main shock [INGV (National Institute of Geophysics and Volcanology) web site].

The Amare cave (L'Aquila) is situated on the southern slope of the Gran Sasso mountain chain, at a distance of $13 \mathrm{~km}$ from the epicentre, in a North/Northwest direction. This chain is one of the largest karst areas of the Italian Apennines and it largely consists of limestone of different geologic epochs. The cave extends for more than one hundred metres from the mouth and reaches a depth of about eighty metres below the ground level. At the bottom of the cave a little (10 $\mathrm{m}$ in diameter) karst pool, called De Marchi pool, is located. Dives exceeding $30 \mathrm{~m}$ were unable to reveal the bottom of this pool and it is likely that this pool is linked with some aquifer of the whole basin that is one of the largest water reserves of central Italy. Near the mouth of the cave a natural spring, named Pietà spring is located. Since 1985, in order to investigate possible earthquakes precursors, some of the authors have installed: a) electromagnetic and acoustic equipments (Bella et al., 1989) near the bottom of the cave, b) a level meter in the De Marchi pool, c) a receiver able to measure the intensity of three LF radio signals: CLT

Published by Copernicus Publications on behalf of the European Geosciences Union. 
( $f=189 \mathrm{kHz}$, Sicily, Italy), MCO ( $f=216 \mathrm{kHz}$, France) and CZE ( $f=270 \mathrm{kHz}$, Czech Republic), in the mouth of the cave. In addition the flow rate of the Pietà spring has been sampled systematically. Many results were obtained in the framework of this research (Bella et al., 1992, 1994a, b, 1996, 1998a, b, c; Biagi and Hayakawa, 2002; Biagi et al., 2001a, b, c, 2002, $2004,2006 a, b, 2008)$. Due to several problems related to the power system, to the data transmission, to different malfunctions of the equipments and so on, practically only the radio signals were collected systematically during the last four years. Moreover, it must be noted that the CLT transmitter switched off the broadcasts in August 2004.

During 2008 a new radio receiver was planned and built up and the old radio receiver operating on the mouth of the Amare cave was substituted with a new one. The result here presented was obtained by means of this receiver.

\section{Radio receiver and measurements network}

The new radio receiver, made by the factory Elettronika (Palo del Colle, Bari, Italy), is shown in the Fig. 1b. The equipment can be powered by batteries or by the main AC power supply. It works in VLF (20-50 kHz) and LF (150-300 kHz) bands monitoring ten frequencies distributed in these bands; for each of them, the apparatus saves the power level detected on a non-volatile memory at a customizable sampling time interval. The receiver must be connected to two different rectilinear antennas (one per band) with the corresponding preamplifier (Fig. 1a) located near the relative antenna. The aim of the preamplifier is to convert the high impedance of the low-frequency antennas in a low impedance to drive the cable and to amplify the captured signal level introducing very low noise. The huge amount of data collected by the receiver is organized in text files, one for each day. The configuration of the equipment, the check of its status and the download of the collected data are possible by a standard, powerful and ubiquitous Ethernet interface. Another main feature is the internal GSM modem that can be used to remotely control the receiver by means of data calls. The interface and the possibilities during remote connection are the same as the local control by Ethernet.

Figure 2 shows the MCO radio signal collected by the old receiver and by the new one in the site described in the previous section. From the Figure inspection it is evident that the running of the two equipments is practically the same; the new receiver is characterized by only a larger dynamic range.

At the end of January 2009, a meeting was organized in Bari (Italy). On this occasion an international cooperation for studying earthquakes precursors was planned (web: http: //beta.fisica.uniba.it/infrep/); in particular, a network consisting of five receivers for sampling LF and VLF radio signals was defined. This network is shown in the map of Fig. 3. The receiver indicated in central Italy is the one substituting

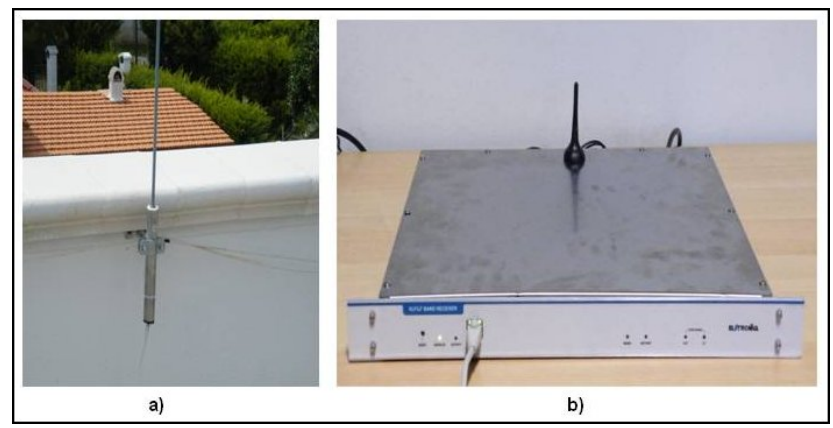

Fig. 1. (a) One antenna with the relative preamplifier connected with the VLF/LF receiver. (b) The radio receiver made by Elettronika factory; the GSM antenna is visible, too.
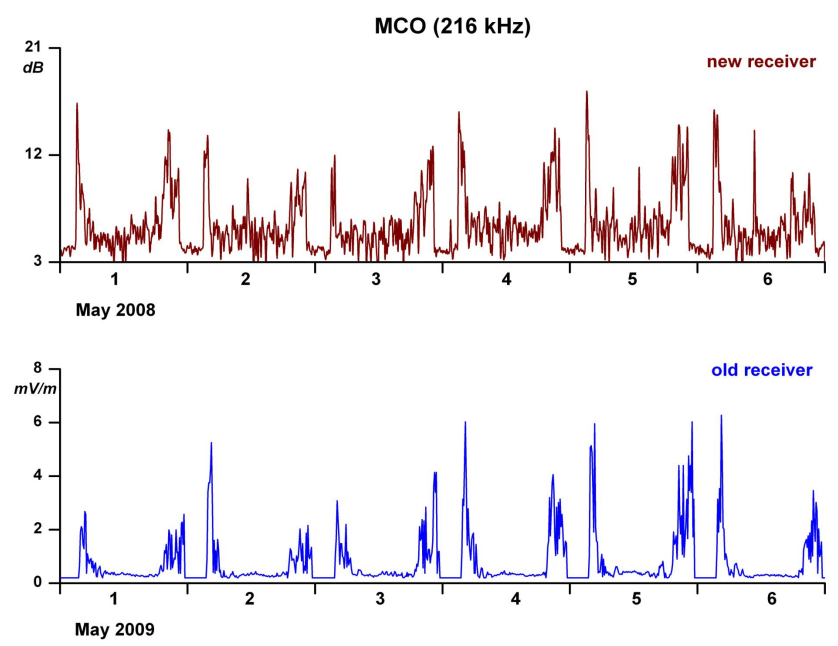

Fig. 2. Intensity of MCO $(216 \mathrm{kHz})$ radio signal collected in central Italy (mouth of the Amare cave, L'Aquila) by means of the new Elettronika receiver (top) and of the old one (bottom). The data of the top trend are related to May 2009; those of the bottom trend to May 2008.

the old receiver at the mouth of the Amare cave. In the map of Fig. 3 also the location of the different VLF/LF transmitters is indicated. During March 2009, the network starts to operate. The data are collected separately by the different teams. The data bank is at the Department of Physics of the University of Bari, that is the headquarter of the network.

\section{Results}

Figure 4 shows the intensity of the five LF radio signals collected by the receiver into operation at the mouth of the Amare cave from the beginning of its running (27 March 2009) up to end of April 2009. The LF transmitters are FRI (162 kHz, France), TRT (180 kHz, Turkey), EU1 (183 kHz, Germany), MCO $(216 \mathrm{kHz}$, France) and CZE $(270 \mathrm{kHz}$, Czech Republic); their location is indicated in Fig. 5. It must 


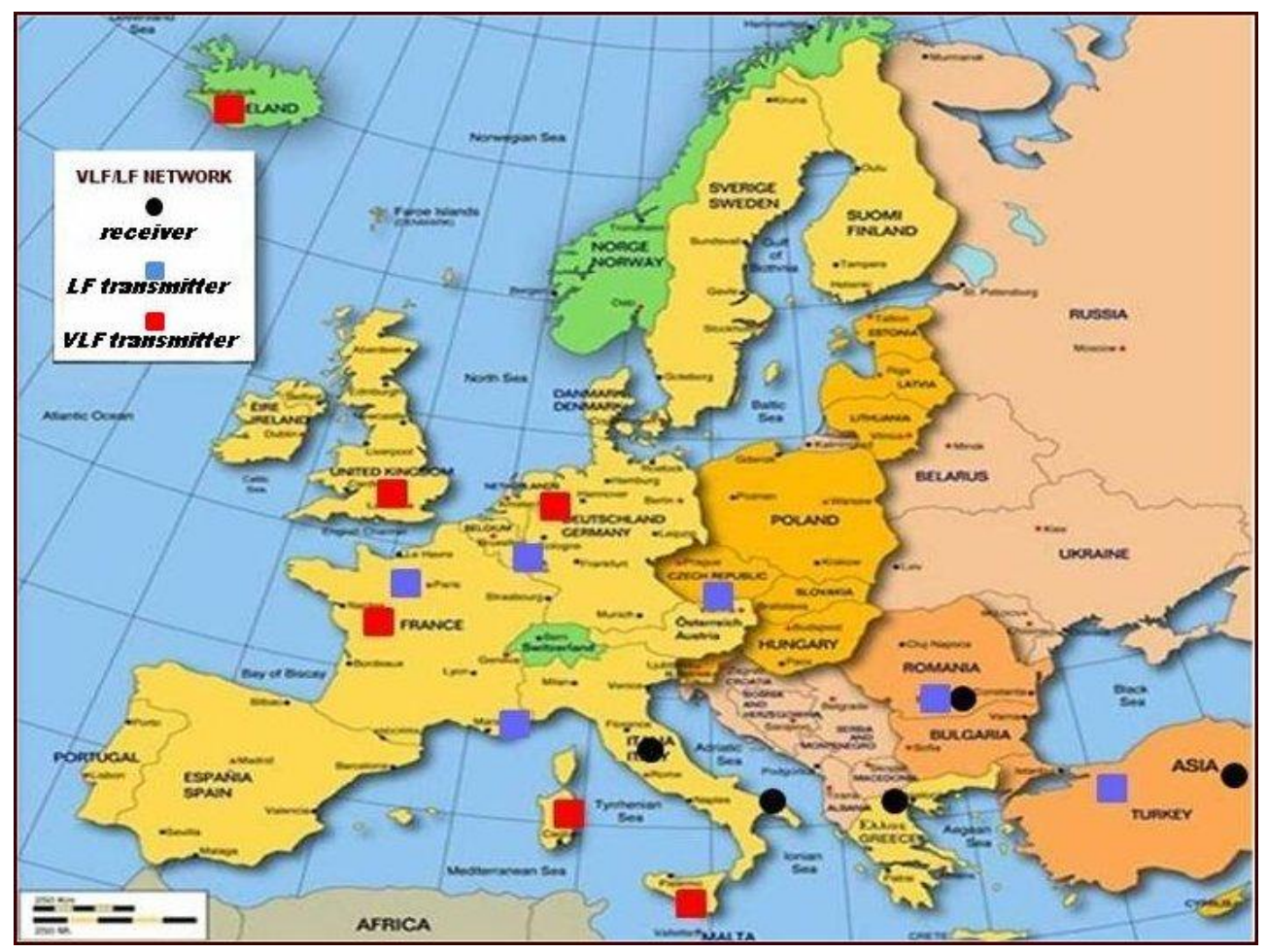

Fig. 3. Map showing the location (circles) of the five VLF/LF receivers into operation. They are located in central Italy (mouth of the Amare cave, L'Aquila), south Italy, Romania, Greece and Turkey. The transmitters, whose signals are sampled by the different receivers, are also indicated (squares).

be noted that: a) the sampling of the FRI signal was set up by remote on 30 March; b) on 4 April, some dB gain of the LF preamplifier was set up by an attendance in loco.

From Fig. 4, a drop of the signal radiated by MCO transmitter from 31 March to 1 April stands up clearly. The feature of the drop can be evaluated taking into account that the mean daily value $(\mathrm{dB})$ of the signal in the normal days preceding the preamplifier gain adjustment is $5.0 \pm 1.7$ while in the previous time interval is $3.8 \pm 0.2$, where the uncertainty indicates the standard deviation. The possibility that this drop could be connected with the transmitter must be excluded because on the same days, this radio signal was normally recorded by the other two receivers (south Italy and Romania) of the measurements network (Fig. 1) operating at that time, as it is shown in Fig. 6. The possibility that the drop could be connected with the receiver was examined and discussed with the engineers of the Elettronika factory and it was excluded also on the basis that the other LF signals are not affected by the drop. So, the only justification of the drop is a defocusing of the MCO radio signal occurred along the path transmitter-receiver. Defocusing of radio signals is well known in radio physics (CCIR Reports, 1990a, b, c) and they are related to ducts, scattering zones, reflecting layers and so on, produced by particular meteorological conditions or synoptic processes such as subsidence, advection, or surface

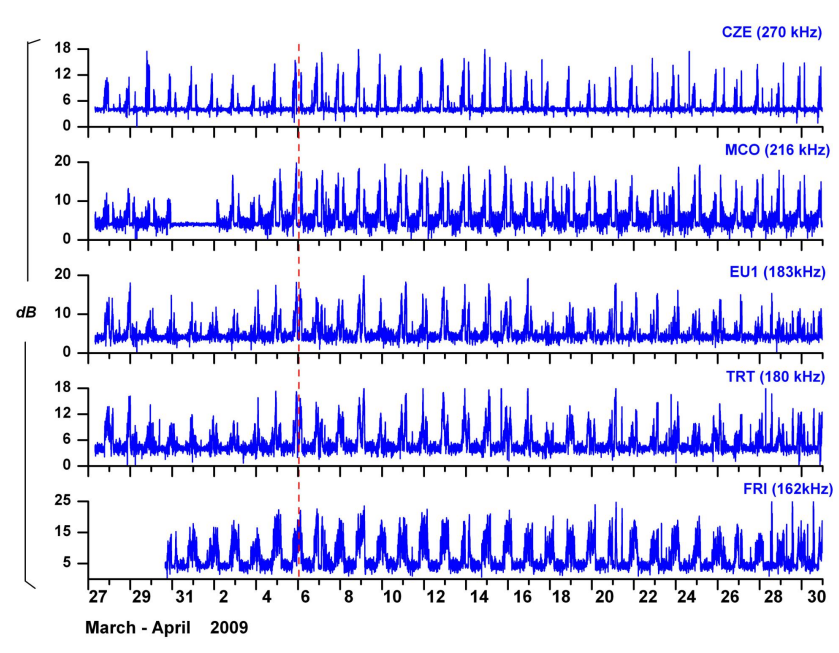

Fig. 4. Intensity of CZE $(270 \mathrm{kHz}), \mathrm{MCO}(216 \mathrm{kHz}), \mathrm{EU} 1$ $(183 \mathrm{kHz})$, TRT $(180 \mathrm{kHz})$ and FRI $(162 \mathrm{kHz})$ radio signals collected by the (Elettronika) receiver located in central Italy (mouth of the Amare cave, L'Aquila) in the period 27 March-30 April 2009. The sampling of the FRI channel started 4 days later. The vertical dashed line indicates the time occurrence of the Abruzzo earthquake. 


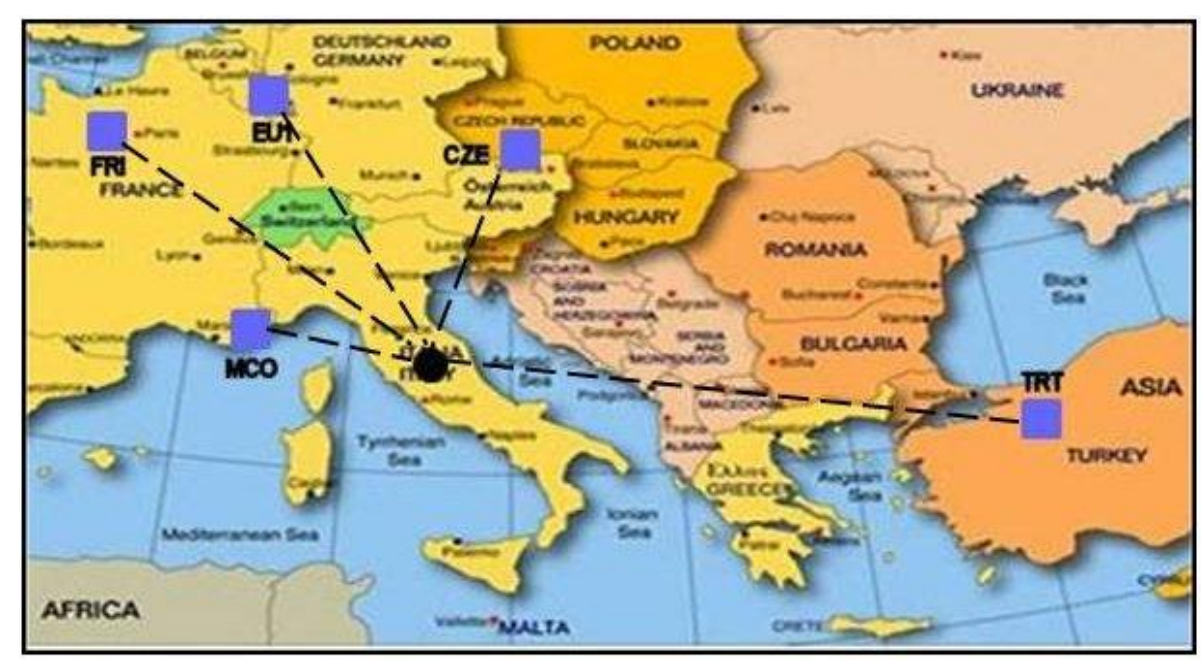

Fig. 5. Map showing the location of the five LF transmitters reported in Fig. 4. The black circle indicates the location of the receiver. The different radio paths are also drawn.

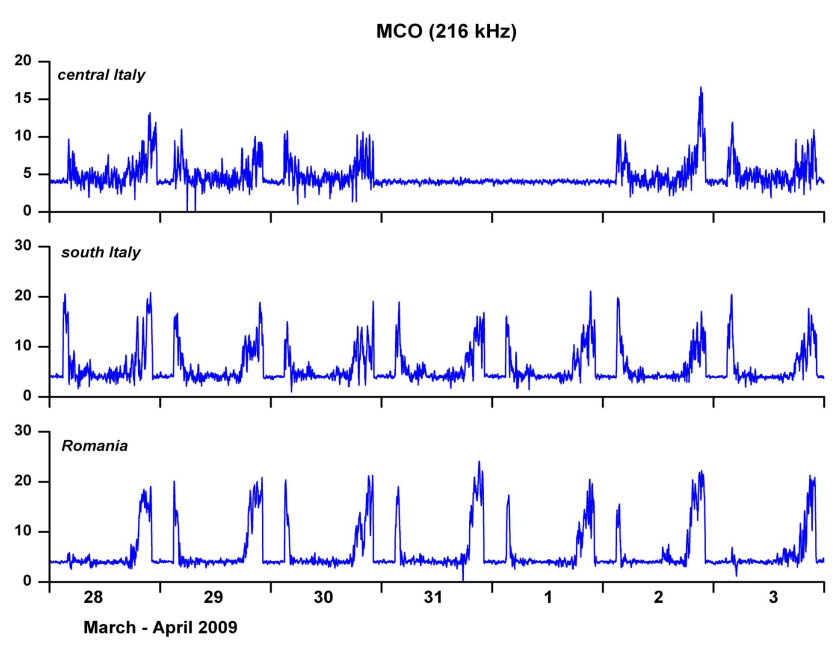

Fig. 6. $\mathrm{MCO}(216 \mathrm{kHz})$ radio signal recorded in central Italy (mouth of the Amare cave, L'Aquila), south Italy and Romania from 28 March to 3 April 2009.

heating or radioactive cooling. These situations during 31 March-1 April, were carefully investigated along the path of the MCO radio signal and no particular situation stood up. So, the defocusing we have presented appears as an anomaly.

\section{Discussion}

The LF signals are characterized by the ground-wave and the sky-wave propagation modes. The ground-wave propagates on the ground and in the lower troposphere and provides a stable signal. On the contrary, the sky-wave is greatly variable between day and night and, at day time, between winter and summer. According to the wave hop propagation the- ory, the sky- wave signal received by an antenna can be considered as a ray starting from the transmitter and reflected one or more times (hops) by the lower ionosphere and by the ground. The MCO radio signal recorded at the mouth of the Amare cave was investigated in detail in a previous study (Biagi et al., 2006b); hence it results that at the time of the anomaly the ground wave practically is the day time signal and the sky wave (one hop) is the night time signal. From Fig. 4 it stands up that the anomaly is represented by the disappearance of the signal at day time and at night time. So, a defocusing of both the ground wave and the sky wave occurred. Assuming a common cause for the defocusing, then the relative processes can happened near the transmitter or near the receiver, that is where the sky wave is in the lower troposphere together with the ground wave. The MCO signal, at the time of the anomaly under study was recorded in Romania and in south Italy (Fig. 3) without any drop as we noted previously; so, the zone near the transmitter can be excluded as source of the defocusing. Therefore, the cause of defocusing must be located in the area of the receiver, that is the area where the Abruzzo earthquake occurred. Thus, the radio anomaly under study appears as a precursor of this earthquake. It must be noted that the LF radio signals collected during 20 years with the old receiver in the same place presented a similar anomaly twice in different signals; the first time the anomaly was related to a seismic sequence occurred nearby the receiver (Bella et al., 1998a) and the second one (Biagi et al., 2004) to the volcanic eruption of the Etna volcano (Sicily, south Italy) happened near the transmitter CLT (\$1). The possible explanation of the defocusing under study is as follows: one of the irregularities (ducts, reflecting layers or scattering zones) mentioned above was produced by the electromagnetic/particles emission, gases emanation and so on, occurred in the preparatory area of the 
Abruzzo earthquake. In such way a defocusing of the radio signals coming to the receiver located in this area could to be produced. The reason of the appearance of the effect only on the MCO $(216 \mathrm{kHz})$ signal can be related to the frequency of the signal or to the direction of the radio path. Generally, the previous irregularities are characterized by a cutoff frequency (CCIR, 1990a). In the case under study no effect appears on the radio signals with greater or smaller frequency than $216 \mathrm{kHz}$, as shown in Fig. 4. So, the frequency does not seem to justify the observed phenomenology and the direction of the path must be claimed. From Fig. 5 it can be noted that the MCO-receiver path is the most aligned in West direction with respect to the other ones and this peculiarity can justify the defocusing of only this radio signal. Probably, during the preparatory phase of the Abruzzo earthquake, different processes have developed in this direction producing one of the irregularities mentioned above in a restricted area (some squared kilometer) of the lower atmosphere crossed by the only MCO signal. Finally, it must be noted that the radio anomaly appeared 7 days before the occurrence of the Abruzzo earthquake, and it is in good agreement with the recent results indicating precursor times of maximum 10 days for the anomalies in radio signals (Hayakawa et al., 1996, 2006; Molchanov and Hayakawa, 1998; Rozhnoi et al., 2005, 2006).

\section{Conclusions}

An anomalies in a LF radio signal occurred prior the Abruzzo earthquake have been presented and discussed. This result is encouraging for research into precursory phenomena because it seems possible to obtain information about the spatial location and the time occurrence of forthcoming earthquakes.

Acknowledgements. This research was partially supported by Foundation of the Cassa di Risparmio di Puglia bank (F.C.R.P., Bari, Italy).

Edited by: M. Hayakawa

Reviewed by: A. P. Nickolaenko and another anonymous referee

\section{References}

Bella, F., Bella, R., Biagi, P. F., Della Monica, G., Ermini, A., Manjgaladze, P., Sgrigna, V., and Zilpimiani, D.: A digital recording system of electromagnetic emissions, Nuovo Cimento 12C, 251259, 1989.

Bella, F., Biagi, P. F., Della Monica, G., Zilpimiani, D., Manjgaladze, P., Pokhotelov, O., Sgrigna, V., Ermini, A., and Liperovsky, $\mathrm{V}$.: Monitoring of natural electromagnetic emissions during moderate seismicity in Central Italy, Fizika Zemli, 112-119, 1992.

Bella, F., Bella, R., Biagi, P. F., Caputo, M., Della Monica, G., Ermini, A., Plastino, W., and Sgrigna, V.: Artificial and natural electromagnetic signals revealed during two years in the
Amare cave (Central Italy), Annali di Geofisica, XXXVII, 4146, 1994a.

Bella, F., Biagi, P. F., Caputo, M., Della Monica, G., Ermini, A., Plastino, W., and Sgrigna, V., Electromagnetic background and preseismic anomalies recorded in the Amare cave (Central Italy), in: Electromagnetic Phenomena Related to Earthquake Prediction, edited by: Hayakawa, M. and Fujinawa, Y., TERRAPUB, Tokyo 1994b.

Bella, F., Biagi, P. F., Caputo, M., Della Monica, G., Ermini, A., Plastino, W., and Sgrigna, V.: Normal and anomalous behaviour of electric, magnetic and seismoacoustic signals recorded in the Amare cave, Annali di Geofisica, XXXIX, 97-100, 1996.

Bella, F., Biagi, P. F., Caputo, M., Cozzi, E., Della Monica, G., Ermini, A., Plastino, W., and Sgrigna, V.: Field strenght variations of LF radiowaves prior to earthquakes in Central Italy, Phys. Earth Planet. Int., 105, 279-286, 1998a.

Bella, F., Biagi, P. F., Caputo, M., Cozzi, E., Della Monica, G., Ermini, A., Plastino, W., and Sgrigna, V.: Aquifer-induced seismicity in the Central Apennines (Italy), Pageoph, 153, 179-194, 1998b.

Bella, F., Biagi, P. F., Caputo, M., Della Monica, G., Ermini, A., Plastino, W., and Sgrigna, V.: Anomalies in different parameters related to the $M=3.9$ Gran Sasso earthquake (1992), Phys. Chem. Earth, 23, 959-963, 1998c.

Biagi, P. F., Piccolo, R., Ermini, A., Martellucci, S., Bellecci, C., Hayakawa, M., Capozzi, V., and Kingsley, S. P.: Possible earthquake precursors revealed by LF radio signals, Nat. Hazards Earth Syst. Sci., 1, 99-104, 2001a,

http://www.nat-hazards-earth-syst-sci.net/1/99/2001/.

Biagi, P. F., Ermini, A., and Kingsley, S. P.: Disturbances in LF radiosignals and the Umbria-Marche (Italy) seismic sequence in 1997-1998, Phys. Chem. Earth, 26, 755-759, 2001 b.

Biagi, P. F., Piccolo, R., Ermini, A., Martellucci, S., Bellecci, C., Hayakawa, M., and Kingsley, S. P.: Disturbances in LF radio-signals as seismic precursors, Annali di Geofisica, 44(5/6), 1011-1019, 2001c.

Biagi, P. F., and Hayakawa, M.: Possible premonitory behaviour of LF radiowaves on the occasion of the Slovenia earthquakes (M=5.2-6.0-5.1) occurred on March-May 1998, in Seismo Electromagnetics, edited by: Hayakawa, M. and Molchanov, O., TERRAPUB, Tokyo, 2002.

Biagi, P. F., Ermini, A., Piccolo, R., Loiacono, D., and Kingsley, S. P.: Electromagnetic signals related to micromovements of limestone blocks: a test in karst caves of Central Italy, in: Seismo Electromagnetics, edited by: Hayakawa, M. and Molchanov, O., TERRAPUB, Tokyo, 2002.

Biagi, P. F., Piccolo, R., Castellana, L., Ermini, A., Martellucci, S., Bellecci, C., Capozzi, V., Perna, G., Molchanov, O., and Hayakawa, M.: Variations in a LF radio signal on the occasion of the recent seismic and volcanic activity in Southern Italy, Phys. Chem. Earth, 29, 551-557, 2004.

Biagi, P. F., Castellana, L., Maggipinto, T., Piccolo, R., Minafra, A., Ermini, A., Martellucci, S., Bellecci, C., Perna, G., Capozzi, V., Molchanov, O. A., and Hayakawa, M.: LF radio anomalies revealed in Italy by the wavelet analysis:Possibile preseismic effects during 1997-1998, Phys. Chem. Earth, 31, 403-408, 2006a.

Biagi, P. F., Castellana, L., Maggipinto, T., Ermini, A., Perna, G., and Capozzi, V.: Electric field strength analysis of 216 and $270 \mathrm{kHz}$ broadcast signals recorded during 9 years, Radio Sci., 
41, RS4013, doi:10.1029/2005RS003296, 2006 b.

Biagi, P. F., Castellana, L., Maggipinto, T., and Ermini, A.: An overview on preseimic anomalies in LF radio signals revealed in Italy by wavelet analysis, Annals of Geophysics, 51, 237-246, 2008.

CCIR: Effects of tropospheric refraction on radio-wave propagation, Propagation in non-ionized media, Annex to Vol. V, Report 718-3, 149-188, 1990a.

CCIR: Attenuation by hydrometeors, in particular precipitation, and other atmospheric particles, Propagation in non-ionized media, Annex to Vol. V, Report 721-3, 226-245, 1990 b.

CCIR: Scattering by precipitation, Propagation in non-ionized media, Annex to Vol. V, Report 882-2, 246-250, 1990c.

Hayakawa, M., Molchanov, O. A., Ondoh, T., and Kawai, E.: The precursory signature effect of the Kobe earthquake on subionospheric VLF signals, J. Comm. Res. Lab., 43, 169-180, 1996.
Hayakawa, M., Ohta, K., Maekawa, S., Yamauchi, T., Ida, Y., Gotoh, T., Yonaiguchi, N., Sasaki, H., and Nakamura, T.: Electromagnetic precursors to the 2004 Mid Niigata Prefecture earthquake, Phys. Chem. Earth, 31, 356-364, 2006.

INGV: National Institute of Geophysics and Volcanology, online available at: http://www.ingv.it, 2009.

Molchanov, O. A. and Hayakawa, M.: Subionospheric VLF signal perturbations possibly related to earthquakes, J. Geophys. Res., 103, 17489-17504, 1998.

Rozhnoi, A. A., Solovieva, M. S., Molchanov, O. A., Hayakawa, M., Maekawa, S., and Biagi, P. F.: Anomalies of LF signal during seismic activity in NovemberDecember 2004, Nat. Hazards Earth Syst. Sci., 5, 657-660, 2005, http://www.nat-hazards-earth-syst-sci.net/5/657/2005/.

Rozhnoi, A. A., Solovieva, M., Molchanov, O., Chebrov, V., Voropaev, V., Hayakawa, M., Maekawa, S., and Biagi, P. F.: Preseismic anomaly of LF signal on the wave path Japan-Kamchatka during November 2004, Phys. Chem. Earth, 31, 422-427, 2006. 